\title{
OPEN Feasibility and beneficial effects of an early goal directed therapy after cardiac arrest: evaluation by conductance method
}

Ole Broch ${ }^{1,2}$, Lars Hummitzsch ${ }^{1,3}$, Jochen Renner ${ }^{4}$, Patrick Meybohm ${ }^{5}$, Martin Albrecht ${ }^{1,3}$, Peter Rosenthal ${ }^{3}$, Ann-Christine Rosenthal ${ }^{3}$, Markus Steinfath ${ }^{1,3}$, Berthold Bein ${ }^{6}$ \& Matthias Gruenewald ${ }^{1,3}$

\begin{abstract}
Although beneficial effects of an early goal directed therapy (EGDT) after cardiac arrest and successful return of spontaneous circulation (ROSC) have been described, clinical implementation in this period seems rather difficult. The aim of the present study was to investigate the feasibility and the impact of EGDT on myocardial damage and function after cardiac resuscitation. A translational pig model which has been carefully adapted to the clinical setting was employed. After $8 \mathrm{~min}$ of cardiac arrest and successful ROSC, pigs were randomized to receive either EGDT (EGDT group) or therapy by random computer-controlled hemodynamic thresholds (noEGDT group). Therapeutic algorithms included blood gas analysis, conductance catheter method, thermodilution cardiac output and transesophageal echocardiography. Twenty-one animals achieved successful ROSC of which 13 pigs survived the whole experimental period and could be included into final analysis. CTnT and LDH concentrations were lower in the EGDT group without reaching statistical significance. Comparison of lactate concentrations between 1 and $8 \mathrm{~h}$ after ROSC exhibited a decrease to nearly baseline levels within the EGDT group ( $1 \mathrm{~h}$ vs $8 \mathrm{~h}: 7.9 \mathrm{vs.} 1.7 \mathrm{mmol} / \mathrm{l}, \mathrm{P}<0.01$ ), while in the noEGDT group lactate concentrations did not significantly decrease. The EGDT group revealed a higher initial need for fluids $(P<0.05)$ and less epinephrine administration $(P<0.05)$ post ROSC. Conductance method determined significant higher values for preload recruitable stroke work, ejection fraction and maximum rate of pressure change in the ventricle for the EGDT group. EGDT after cardiac arrest is associated with a significant decrease of lactate levels to nearly baseline and is able to improve systolic myocardial function. Although the results of our study suggest that implementation of an EGDT algorithm for post cardiac arrest care seems feasible, the impact and implementation of EGDT algorithms after cardiac arrest need to be further investigated.
\end{abstract}

In Europe, 150,000-400,000 people per year suffer out of hospital cardiac arrest ${ }^{1}$. The number of patients with return of spontaneous circulation (ROSC) varies between 27 and $61 \%{ }^{2,3}$.

It must be noted, however, that initial ROSC alone is not decisive for patient's survival. Beside successful cardiopulmonary resuscitation, establishment and maintenance of stable hemodynamic conditions are further important targets in the post ROSC time period. However, despite ROSC and intensive therapy, 50-70\% of the patients die in the post-resuscitation period $^{4-6}$. Factors like hyperoxaemia, hyperglycaemia, hypocapnia, hypotension and fever appear to have a significant negative effect on patient's outcome ${ }^{7-9}$. To avoid mismatch of oxygen supply and consumption in the post-cardiac arrest period, hemodynamic stabilization by individually titrated volume and/or catecholamine therapy plays another important role. In this context, an early goal directed therapy (EGDT) seems to have potential beneficial impact on post-cardiac arrest syndrome ${ }^{10,11}$. However, most

${ }^{1}$ Department of Anesthesiology and Intensive Care Medicine, University Hospital Schleswig-Holstein, Campus Kiel, Kiel, Germany. 'Department of Anesthesiology and Intensive Care Medicine, Elbe Hospital Stade, Stade, Germany. ${ }^{3}$ Christian-Albrechts-University Kiel, Kiel, Germany. ${ }^{4}$ Department of Anesthesiology and Intensive Care Medicine, Städtisches Krankenhaus Kiel, Kiel, Germany. ${ }^{5}$ Department of Anesthesiology, Intensive Care, Emergency and Pain Medicine, University Hospital Würzburg, Würzburg, Germany. ${ }^{6}$ Department of Anesthesiology and Intensive Care Medicine, Asklepios Hospital St. Georg, Hamburg, Germany. ${ }^{\boxplus}$ email: lars.hummitzsch@ uksh.de 
of the mentioned studies performed EGDT after cardiac arrest in association with a therapy bundle containing other therapeutic interventions. Moreover, post-resuscitation EGDT was compared to a historical control group which received none of these therapies. Recent studies indicate that early hemodynamic stabilization towards higher blood pressure values is associated with smaller myocardial damage and increasing survival rates after cardiac arrest ${ }^{10,12-14}$.

The aim of our prospective animal study was therefore to investigate the feasibility and the impact of an established EGDT algorithm on myocardial damage and function after cardiac resuscitation compared to a noEGDT group. We evaluated effects of EGDT by established markers of ischemia and myocardial function by advanced hemodynamic monitoring, including the conductance method. cTnT and lactate concentrations were defined as primary outcome parameters, while hemodynamics represented secondary outcome parameters.

We hypothesized that an EGDT based on variables like mean arterial pressure (MAP), central venous pressure $(\mathrm{CVP})$, ejection fraction (EF) and central venous oxygen saturation $\left(\mathrm{S}_{\mathrm{cv}} \mathrm{O}_{2}\right)$ after cardiac arrest is in principle feasible and leads to an improved myocardial function.

\section{Methods}

The study was approved by the local Animal Investigation Committee, Christian-Albrechts-University Kiel, Ministry for Agriculture, Environment and Rural Areas, Schleswig-Holstein (Permit Number: V 312-72241.12139(3-1/10)). The present animal investigation was conducted in consideration of the National Institutes of Health guide for the care and use of Laboratory animals (NIH Publications No. 8023, revised 1978) and complied with the ARRIVE guidelines.

Thirty german domestic pigs with a weight between 28 and $34 \mathrm{~kg}$ were included. Preparation of the animals and experimental setting were based on the protocols described previously ${ }^{15-17}$. Briefly, after fasting overnight with free access to water, the animals were premedicated with the neuropleptic azaperone $(4 \mathrm{mg} / \mathrm{kg})$, $\mathrm{ketamine}$ $(1.0 \mathrm{mg} / \mathrm{kg})$ and atropine $(10 \mu \mathrm{g} / \mathrm{kg}) 1 \mathrm{~h}$ before surgery. Thereafter, propofol $(2 \mathrm{mg} / \mathrm{kg})$ and sufentanil $(0.5 \mu \mathrm{g} /$ $\mathrm{kg}$ ) were administered through a venous access, typically placed in an ear vein, followed by endotracheal intubation. General anaesthesia was performed with the aim of avoiding pain and minimizing distress or suffering for the animals. Pigs were ventilated with the Sulla $808-\mathrm{V}$ ventilator (Dräger AG, Lübeck, Germany) in a volumecontrolled mode with a tidal volume of $8 \mathrm{ml} / \mathrm{kg}$, a positive end-expiratory pressure of $5 \mathrm{~cm} \mathrm{H}_{2} \mathrm{O}$, an I:E ratio of 1:1.5 and $\mathrm{FiO}_{2}$ of 0.4 . Respiratory rate was adjusted to achieve normocapnia ( $\mathrm{pCO}_{2} 35-40 \mathrm{mmHg}$ ). A pulse oximeter was placed on the ear to monitor oxygen saturation (M-CaiOV, Datex-Ohmeda, Helsinki, Finland) and cardiac rhythm was monitored by standard 5-channel electrocardiogram. Anesthesia was maintained by continuous infusion of propofol $(4-8 \mathrm{mg} / \mathrm{kg} / \mathrm{h})$ and sufentanil $(0.3 \mu \mathrm{g} / \mathrm{kg} / \mathrm{h})$. We repeatedly performed pain stimuli like tail clamping and focused on the corneal reflex and lacrimation to detect an inadequate depth of anaesthesia. If necessary, additional sufentanil and propofol was injected. To maintain temperature between 37.0 and $38.0^{\circ} \mathrm{C}$ a heating blanket was used. During the whole study period, the pigs received an infusion of cristalloid solution (Sterofundin, B. Braun-Melsungen AG, Germany) with a flow rate of $10 \mathrm{ml} / \mathrm{kg} / \mathrm{h}$ and an antibiotic prophylaxis with cefuroxime.

Under sterile conditions, a 7 Fr triple lumen central venous catheter (Arrow International, Inc. Reading, PA, USA) was inserted percutaneously in the left internal jugular vein. Thereafter, a 7.5 Fr introducer was placed in the right jugular vein for insertion of a temporary catheter for electrical induction of ventricular fibrillation. Subsequently, a 4.0 Fr thermistor tipped catheter for thermodilution and pulse contour analysis was inserted percutaneously into the femoral artery (PV 2015L20, Pulsiocath, Pulsion Medical Systems AG, Munich, Germany). Additionally, surgical preparation of the right femoral vein was carried out and a $14 \mathrm{Fr}$ introducer for insertion of an occlusion catheter (Fogarty, 120806F Edwards Lifescience, Irvine, CA) was placed into the vein. The right carotid artery was also surgically uncovered for placement of an $8 \mathrm{Fr}$ introducer followed by a 7 Fr conductance catheter (CD Leycom, CA-71103-PL, Hengelo, The Netherlands). A transesophageal echocardiography probe was firstly inserted to control the position of the conductance catheter and, secondly, for measurement of EF by the simpson's method of discs.

After connection to the PiCCOplus Monitoring system (Version 6.0, Pulsion Medical Systems AG, Munich, Germany), this system allows discontinuous measurement of $\mathrm{CO}_{\text {TPTD }}$ by transpulmonary thermodilution and other hemodynamic variables, measurement of $\mathrm{CVP}, \mathrm{S}_{\mathrm{cv}} \mathrm{O}_{2}$, transpulmonary thermodilution cardiac output $\left(\mathrm{CO}_{\mathrm{TPTD}}\right)$ and stroke volume $\left(\mathrm{SV}_{\mathrm{TPTD}}\right)$ was derived from central venous catheter and PiCCO catheter, respectively. For assessment of $\mathrm{CO}_{\mathrm{TPTD}}$ and $\mathrm{SV}_{\mathrm{TPTD}}$, thermodilution measurements were obtained by injecting $10 \mathrm{ml}$ ice cold saline $\left(\leq 8^{\circ} \mathrm{C}\right)$ through the central venous catheter. Without taking account of the respiratory cycle, injections were performed at least three times. In case of a difference of $\mathrm{CO}_{\text {TPTD }} \geq 15 \%$, preceding measurement was discarded and calibration repeated. Transesophageal echocardiography for evaluation of EF was performed by a single experienced examiner using a multiplane transesophageal echocardiography probe (GE Healthcare $6 \mathrm{~T}$ TEE probe, Vivid i-System, Wauwatosa USA). Calculations by the conductance catheter method (CD Leycom Sigma, Zoetermeer, The Netherlands) included left ventricular end-systolic elastance (Ees), preload recruitable stroke work (PRSW, stroke work-to-end-diastolic volume relationship), diastolic compliance (EDPVR, enddiastolic pressure-volume relation) and a parameter of early diastolic relaxation (Tau). For determination of preload-independent parameters during the measurements by conductance method, an occlusion catheter was placed in the inferior vena cava and inflated for 5-10 s.

After instrumentation, all respiratory, hemodynamic and echocardiographic data recordings were performed at baseline. Arterial and central venous blood samples were collected for blood gas analysis (GEM Primer 4000, Werfen Germany, Munich). All measurements were performed with the animal in supine position. The experimental protocol is illustrated in Fig. 1. 


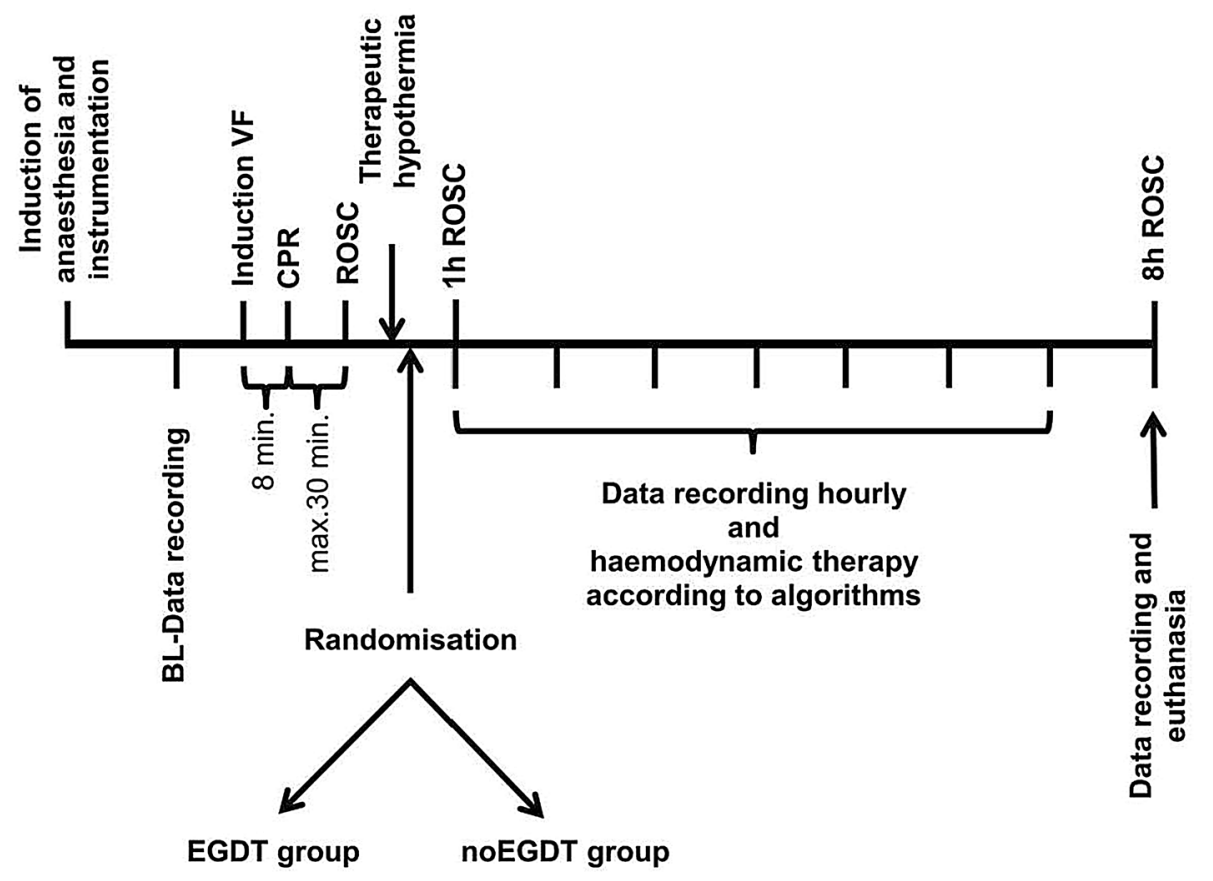

Figure 1. Illustration of experimental protocol. Data collection was performed at baseline and hourly after ROSC. $B L$ baseline, $V F$ ventricular fibrillation, $C P R$ cardiopulmonary resuscitation, $R O S C$ return of spontaneous circulation, EGDT early goal directed therapy.

To prevent any blood clotting caused by the placed catheters, pigs received $100 \mathrm{IE} / \mathrm{kg}$ heparin. Thereafter, a temporary catheter for electrical induction of ventricular fibrillation was advanced through the introducer in the right jugular vein. After induction of ventricular fibrillation, mechanical ventilation was stopped and cardiopulmonary resuscitation was started after $8 \mathrm{~min}$ of cardiac arrest in accordance to the guidelines from the European resuscitation council ${ }^{18}$. For the first $4 \mathrm{~min}$ basic life support was carried out with a chest compression of 100/min and a relationship of compression-to-ventilation of 30:2. Advanced cardiac life support was initiated by one biphasic defibrillation using $4 \mathrm{~J} / \mathrm{kg}$ (Corpuls 3 (ED 530), Kaufering, Germany). Besides continuous chest compression further ventilation was performed with $100 \%$ oxygen and an initial respiratory rate of $20 / \mathrm{min}$. All pigs received $5 \mathrm{mg} / \mathrm{kg}$ amiodarone and intermittent every $2 \mathrm{~min} 15 \mu \mathrm{g} / \mathrm{kg}$ epinephrine or $0.4 \mathrm{IE} / \mathrm{kg}$ vasopressin. Cardiopulmonary resuscitation was stopped if ROSC was observed which was defined as maintenance of an unassisted pulse and a systolic aortic blood pressure of $\geq 60 \mathrm{mmHg}$ lasting for ten consecutive minutes according to the Utstein-style guidelines ${ }^{19}$. If resuscitation remained unsuccessful after $30 \mathrm{~min}$, cardiopulmonary resuscitation was terminated. After ROSC, a mild therapeutic hypothermia was initiated in all animals to achieve a temperature ranging between 32 and $34^{\circ} \mathrm{C}$ and inspiratory oxygen concentration was reduced. Just before the end of the first hour after ROSC, animals were randomized either into EGDT or noEGDT group. Subsequently, measurements by conductance method, transpulmonary thermodilution via PiCCO catheter and transesophageal echocardiography were performed every hour after ROSC. Additionally, arterial and central venous blood gas analyses were carried out. Blood samples for quantification of cardiac troponin $\mathrm{T}$ (cTnT), lactate and lactate dehydrogenase $(\mathrm{LDH})$ were obtained at various time points after ROSC. After completion of the measurements, a therapy algorithm was established and hemodynamic therapy was conducted in dependence of group allocation. The core variables, MAP, CVP, EF and $\mathrm{S}_{\mathrm{cv}} \mathrm{O}_{2}$ were evaluated in a fixed and predefined order. Therapeutic options were limited to a targeted repetitive volume administration (bolus of $250 \mathrm{ml}$ balanced crystalloids) and the targeted adjustment (stepwise $1-10 \mu \mathrm{g} / \mathrm{min}$ ) of vasopressors (norepinephrine) or inotropes (epinephrine). Hemodynamic therapy and target variables were similar but threshold values differed between both groups. In the EGDT group, target area was MAP $>80 \%$ of the baseline, $\mathrm{CVP}>7 \mathrm{mmHg}, \mathrm{EF}>60 \%$ and $\mathrm{S}_{\mathrm{cv}} \mathrm{O}_{2}>70 \%$. In the noEGDT group, thresholds of target variables were defined by a stochastic random model on an hourly basis. Thresholds could be equal, higher or lower compared to the EGDT group. Limit ranges in the noEGDT group were defined as MAP 55-120\% of the baseline, CVP 2-8 mmHg, EF 40-70\% and $\mathrm{S}_{\mathrm{cv}} \mathrm{O}_{2} 50-80 \%$ (Supplement 1 ). The detailed therapy algorithm is displayed in Fig. 2. For both groups intervention thresholds indicating direct vital threat were defined as drop of $<40 \%$ of baseline for MAP and $<45 \%$ for $\mathrm{S}_{\mathrm{cv}} \mathrm{O}_{2}$. If values fell below the set threshold, individual therapeutic measures were initiated by an experienced physician independently from the therapy algorithm.

Therapy was stopped if pre-defined targets were achieved or at the start of a new period of measurements. Latest therapeutic algorithm was initiated after seven hours and ended after data recording eight hours after ROSC. After completion of the trial period, animals were euthanized by an overdose of sufentanil, propofol and potassium chloride. 


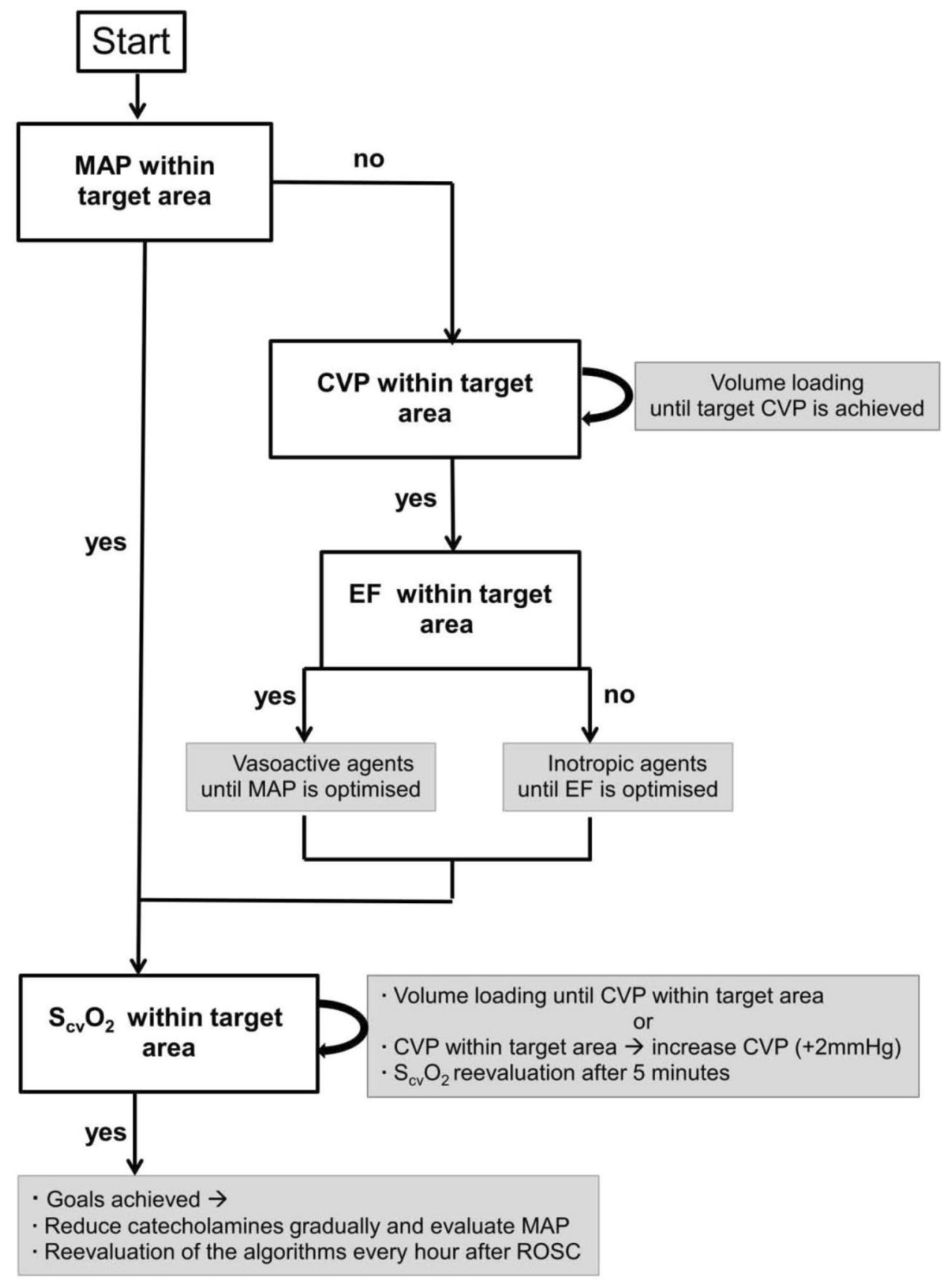

Figure 2. Therapy algorithm.

Statistical analysis. Statistical comparisons were performed using commercially available statistics software (GraphPad Prism 6, Version 6.03, GraphPad Software Inc., San Diego, CA, USA). To determine the distribution between the population and the variables studied, a Shapiro-Wilk test was performed. Pooled data of both groups were compared by unpaired t-test (normally distributed) or Mann-Whitney-U-Test (not normally distributed). In case of normally distributed data, two-way ANOVA was performed for analysis of repeated measurements over the period of time. Occasional missing data in the time kinetics were filled by the "multiple imputation technique" (MIT) which is available in the STATA 13 statistics software (StataCorp LP, CollegeStation, TX, USA). To evaluate the effect of hemodynamic therapy, variables at one and eight hours after ROSC were compared by paired $t$-test or Wilcoxon sign rank test. All variables are expressed as mean \pm SD or median [25-75\% quartiles].

Ethics approval. The study was approved by the local Animal Investigation Committee, Christian-Albrechts-University Kiel, Ministry for Agriculture, Environment and Rural Areas, Schleswig-Holstein (Permit 


\begin{tabular}{|l|c|l|l|}
\hline & EGDT $(\mathrm{N}=7)$ & noEGDT (N =6) & P-value \\
\hline Weight $(\mathrm{kg})$ & $32 \pm 2.4$ & $32 \pm 2.7$ & 0.76 \\
\hline Duration CPR $(\mathrm{min})$ & $10 \pm 3$ & $10 \pm 8$ & 0.38 \\
\hline Cumulative energy $(\mathrm{J})$ & $656 \pm 328$ & $667 \pm 621$ & 0.70 \\
\hline Epinephrine during CPR $(\mu \mathrm{g})$ & $1350 \pm 645$ & $828 \pm 497$ & 0.18 \\
\hline Amiodarone during CPR $(\mathrm{mg})$ & $150 \pm 43$ & $171 \pm 104$ & 0.85 \\
\hline Vasopressin during CPR $(\mathrm{IE})$ & $17 \pm 10$ & $18 \pm 8$ & 0.99 \\
\hline
\end{tabular}

Table 1. Descriptive data. Values are presented as mean \pm SD. CPR cardiopulmonary resuscitation.

\begin{tabular}{|c|c|c|c|}
\hline & EGDT $(N=7)$ & noEGDT $(\mathrm{N}=6)$ & P-value \\
\hline HR (bpm) & $100 \pm 19$ & $101 \pm 19$ & 0.86 \\
\hline MAP (mmHg) & $86 \pm 19$ & $99 \pm 15$ & 0.22 \\
\hline CVP (mmHg) & $4.0 \pm 3.0$ & $4.0 \pm 2.0$ & 0.60 \\
\hline $\mathrm{CO}_{\text {TPTD }}(\mathrm{l} / \mathrm{min})$ & $4.2 \pm 0.7$ & $4.6 \pm 0.8$ & 0.35 \\
\hline $\mathrm{EF}(\%)$ & $67 \pm 10$ & $62 \pm 6$ & 0.29 \\
\hline $\mathrm{SV}^{\mathrm{c}}(\mathrm{ml})$ & $59 \pm 18$ & $64 \pm 13$ & 0.65 \\
\hline $\operatorname{Ees}^{\mathrm{c}}(\mathrm{mmHg} / \mu \mathrm{l})$ & $1.9 \pm 1.1$ & $1.5 \pm 1.4$ & 0.42 \\
\hline $\mathrm{PRSW}^{\mathrm{c}}(\mathrm{mmHg})$ & $76 \pm 20$ & $64 \pm 22$ & 0.42 \\
\hline $\operatorname{EDPVR}^{\mathrm{c}}(\mathrm{mmHg} / \mu \mathrm{l})$ & $0.3 \pm 0.1$ & $0.3 \pm 0.1$ & 0.75 \\
\hline $\mathrm{Tau}^{\mathrm{c}}(\mathrm{ms})$ & $40 \pm 26$ & $24 \pm 9$ & 0.27 \\
\hline $\mathrm{pH}$ & $7.5 \pm 0.1$ & $7.5 \pm 0.0$ & 0.28 \\
\hline $\mathrm{S}_{\mathrm{cv}} \mathrm{O}_{2}(\%)$ & $78 \pm 8$ & $72 \pm 9$ & 0.23 \\
\hline Lactate $(\mathrm{mm} / \mathrm{l})$ & $1.4 \pm 0.8$ & $1.6 \pm 0.8$ & 0.71 \\
\hline cTNT $(\mathrm{pg} / \mathrm{ml})$ & $47 \pm 91$ & $3.0 \pm 1.0$ & 0.15 \\
\hline
\end{tabular}

Table 2. Hemodynamic variables and laboratory parameters at baseline. Values are presented as mean \pm SD. $H R$ heart rate, $M A P$ mean arterial pressure, $C V P$ central venous pressure, $C O_{T P T D}$ cardiac output derived from transpulmonary thermodilution, $E F$ ejection fraction, $S V$ stroke volume, Ees end-systolic elastance, $P R S W$ preload recruitable stroke work, EDPVR end-diastolic pressure-volume relation, Tau early diastolic relaxation, $\mathrm{S}_{\mathrm{CV}} \mathrm{O}_{2}$ central venous oxygen saturation, $c T N T$ cardiac troponin $\mathrm{T},{ }^{c}$ variables measured by conductance catheter.

Number: V 312-72241.121-39(3-1/10)). The present animal investigation was conducted in consideration of the National Institutes of Health guide for the care and use of Laboratory animals (NIH Publications No. 8023, revised 1978) and complied with the ARRIVE guidelines.

\section{Results}

A total of 30 pigs were investigated from which 21 achieved ROSC after 8 min of cardiac arrest. Two animals died within the first hour after ROSC due to intra-abdominal bleedings, so that 19 pigs were randomly assigned to each group (EGDT group, $\mathrm{N}=10$; noEGDT group, $\mathrm{N}=9$ ). Subsequently, three animals of each group died, so that seven animals in the EGDT group and six animals in the noEGDT group could be included into the final analysis. One animal in the EGDT group showed already marked increase in cTNT levels at baseline. Descriptive and resuscitation data are presented in Table 1 . Note that the standard deviation but not the mean of the duration of cardiopulmonary resuscitation differed notably between both groups and was shifted towards shorter times in the control group (noEGDT).

Hemodynamic and laboratory variables at baseline are shown in Table 2. There were no significant differences between both groups.

The hourly control of the target variables led to the implementation of the therapeutic algorithm (MAP and/ or $\mathrm{S}_{\mathrm{CV}} \mathrm{O}_{2}$ not in target range) in total 47 times in the EGDT group (average of 6.7 per animal) and 42 times (average of 7 per animal) in the noEGDT group. The hemodynamic algorithm cycles were more often successfully completed within EGDT group (83\% of attempts) compared to noEGDT group (71\% of attempts). In the EGDT group, targeted values could be reached in 42 of 47 therapeutic algorithms (85\%) and in 31 of 42 algorithms for the noEGDT group (74\%). Therapeutic algorithm required $18 \mathrm{~min}( \pm 14)$ in the EGDT group and $19 \mathrm{~min}( \pm 17)$ in the noEGDT group by average. In the noEGDT group, MAP fell twice considerably below life-threatening threshold values and needed further rescue intervention. An overview of cardiac variables and $\mathrm{S}_{\mathrm{cv}} \mathrm{O}_{2}$ for EGDT and noEGDT group at different time points is shown in Table 3. Pooled data revealed significant differences for EF, PRSW, EDV and $\mathrm{dp} / \mathrm{dt}_{\max }$ between animals in the EGDT and noEGDT group. Statistically significant differences between the EGDT and the noEGDT group were also observed for the following parameters and time 


\begin{tabular}{|c|c|c|c|c|c|c|c|c|c|c|}
\hline & \multicolumn{2}{|l|}{ BL } & \multicolumn{2}{|l|}{1 h ROSC } & \multicolumn{2}{|l|}{$4 \mathrm{~h}$ ROSC } & \multicolumn{2}{|l|}{$8 \mathrm{~h}$ ROSC } & \multicolumn{2}{|l|}{ Pooled data } \\
\hline & EGDT & No EGDT & EGDT & No EGDT & EGDT & No EGDT & EGDT & No EGDT & EGDT & No EGDT \\
\hline MAP (mmHg) & $86 \pm 19$ & $99 \pm 15$ & $71 \pm 13$ & $77 \pm 12$ & $79 \pm 27$ & $80 \pm 8$ & $89 \pm 34$ & $76 \pm 14$ & $79 \pm 7$ & $80 \pm 7$ \\
\hline $\mathrm{SV}^{\mathrm{c}}(\mathrm{ml})$ & $59 \pm 18$ & $64 \pm 13$ & $47 \pm 11$ & $49 \pm 19$ & $42 \pm 14$ & $45 \pm 19$ & $38 \pm 8$ & $50 \pm 13$ & $45 \pm 6$ & $49 \pm 6$ \\
\hline $\mathrm{EF}(\%)$ & $67 \pm 10$ & $62 \pm 6$ & $62 \pm 11$ & $56 \pm 10$ & $58 \pm 7$ & $43 \pm 17$ & $52 \pm 6$ & $46 \pm 15$ & $57 \pm 5$ & $51 \pm 7^{*}$ \\
\hline PRSW $^{c}(\mathrm{mmHg})$ & $76 \pm 20$ & $64 \pm 22$ & $55 \pm 17$ & $52 \pm 6$ & $57 \pm 8$ & $50 \pm 6$ & $68 \pm 20$ & $37 \pm 12^{\# \#}$ & $64 \pm 8$ & $49 \pm 7^{*}$ \\
\hline $\mathrm{Ees}^{\mathrm{c}}(\mathrm{mmHg} / \mathrm{ml})$ & $1.9 \pm 1.0$ & $1.5 \pm 1.4$ & $0.8 \pm 0.2$ & $1.1 \pm 0.6$ & $1.5 \pm 0.7$ & $1.4 \pm 0.7$ & $1.6 \pm 1.1$ & $0.8 \pm 0.5$ & $1.5 \pm 0.3$ & $1.3 \pm 0.3$ \\
\hline EDPVR $^{c}(\mathrm{mmHg})$ & $0.3 \pm 0.1$ & $0.3 \pm 0.1$ & $0.3 \pm 0.1$ & $0.3 \pm 0.1$ & $0.3 \pm 0.1$ & $0.5 \pm 0.2^{*}$ & $0.4 \pm 0.1$ & $0.5 \pm 0.2$ & $0.3 \pm 0.0$ & $0.4 \pm 0.1$ \\
\hline $\mathrm{EDV}^{\mathrm{c}}(\mathrm{ml})$ & $78 \pm 21$ & $102 \pm 18$ & $71 \pm 22$ & $85 \pm 24$ & $77 \pm 23$ & $92 \pm 33$ & $69 \pm 29$ & $113 \pm 24^{\# \#}$ & $73 \pm 10$ & $99 \pm 14^{*}$ \\
\hline $\mathrm{EDP}^{\mathrm{c}}(\mathrm{mmHg})$ & $21 \pm 16$ & $14 \pm 5$ & $18 \pm 14$ & $12 \pm 11$ & $9 \pm 13$ & $27 \pm 22$ & $18 \pm 4$ & $21 \pm 7$ & $16 \pm 6$ & $22 \pm 7$ \\
\hline $\mathrm{Dp} / \mathrm{dt}_{\max }{ }^{\mathrm{c}}(\mathrm{mmHg} / \mathrm{s})$ & $2866 \pm 1208$ & $2445 \pm 531$ & $4054 \pm 545$ & $3611 \pm 662$ & $5083 \pm 1265$ & $2912 \pm 1231^{*}$ & $3991 \pm 2746$ & $2052 \pm 786$ & $4452 \pm 751$ & $2879 \pm 644^{*}$ \\
\hline $\mathrm{Dp} / \mathrm{dt}_{\min }{ }^{\mathrm{c}}(\mathrm{mmHg} / \mathrm{s})$ & $-1869 \pm 992$ & $-2734 \pm 826$ & $-1557 \pm 335$ & $-1909 \pm 367$ & $-1890 \pm 647$ & $-1755 \pm 1011$ & $-1964 \pm 947$ & $-2014 \pm 472$ & $-1845 \pm 181$ & $-2033 \pm 280$ \\
\hline $\operatorname{Tau}^{\mathrm{c}}(\mathrm{ms})$ & $40 \pm 26$ & $24 \pm 9$ & $34 \pm 19$ & $22 \pm 4$ & $32 \pm 27$ & $37 \pm 20$ & $27 \pm 11$ & $26 \pm 6$ & $31 \pm 5$ & $28 \pm 5$ \\
\hline $\mathrm{S}_{\mathrm{CV}} \mathrm{O}_{2}(\%)$ & $78 \pm 8$ & $72 \pm 9$ & $80 \pm 9$ & $82 \pm 9$ & $77 \pm 7$ & $67 \pm 12$ & $72 \pm 13$ & $59 \pm 12$ & $75 \pm 5$ & $70 \pm 7$ \\
\hline $\mathrm{CO}_{\mathrm{TPTD}}(\mathrm{l} / \mathrm{min})$ & $4.2 \pm 0.7$ & $4.6 \pm 0.8$ & $4.6 \pm 1.9$ & $4.8 \pm 1.7$ & $4.3 \pm 1.8$ & $4.0 \pm 1.6$ & $4.8 \pm 0.7$ & $4.5 \pm 1.2$ & $4.5 \pm 1.4$ & $4.5 \pm 1.3$ \\
\hline SSVRI $\left(\operatorname{dyn}^{*} \mathrm{~s}^{*} \mathrm{~cm}^{-5 *} \mathrm{~m}^{2}\right)$ & $1766 \pm 376$ & $1656 \pm 608$ & $1350 \pm 724$ & $1308 \pm 539$ & $1572 \pm 965$ & $1737 \pm 716$ & $1393 \pm 739$ & $1308 \pm 359$ & $1505 \pm 726$ & $1500 \pm 555$ \\
\hline
\end{tabular}

Table 3. Hemodynamic variables and parameters of myocardial function during the study period. Values are presented as mean $\pm \mathrm{SD}$. MAP mean arterial pressure, $S V$ stroke volume, $E F$ ejection fraction, $P R S W$ preload recruitable stroke work, Ees end-systolic elastance, $E D P V R$ end-diastolic pressure-volume relation, $E D V$ end-diastolic volume, $E D P$ end-diastolic pressure, $D p / d t_{\max }$ maximal rate of rise of left ventricular pressure, $\mathrm{Dp} / d t_{\min }$ minimal rate of rise of left ventricular pressure, Tau early diastolic relaxation, $\mathrm{S}_{\mathrm{CV}} \mathrm{O}_{2}$ central venous oxygen saturation, $\mathrm{CO}_{T P T D}$ cardiac output derived from transpulmonary thermodilution, $S V R I$ systemic vascular resistance index, ${ }^{c}$ variables measured by conductance catheter. ${ }^{\#} \mathrm{P}<0.05 ;{ }^{\# \#} \mathrm{P}<0.01$ (repeated measures two-way ANOVA); ${ }^{*} \mathrm{P}<0.05$ (unpaired t-test or Mann-Whitney-U-Test).
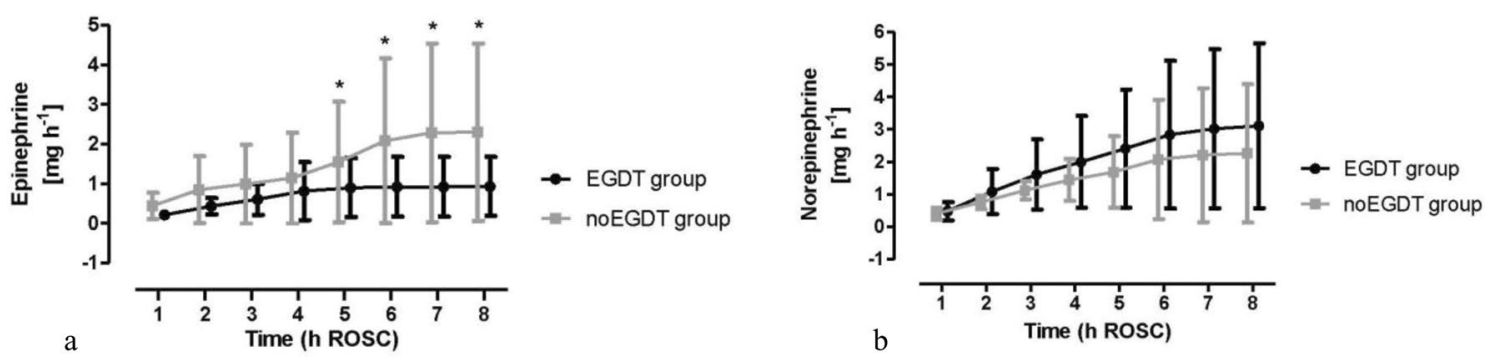

Figure 3. Cumulative consumption of epinephrine (a) and norepinephrine (b). ROSC return of spontaneous circulation. Bars denote SD.

points: PRSW ( $8 \mathrm{~h}$ after ROSC, $\mathrm{P}<0.01$ ), EDPVR ( $4 \mathrm{~h}$ after ROSC, $\mathrm{P}<0.05$ ), EDV ( $8 \mathrm{~h}$ after ROSC, $\mathrm{P}<0.01)$ and $\mathrm{Dp} / \mathrm{dt}_{\max }$ (4 h after ROSC, $\mathrm{P}<0.05$; Table 3 ).

The total amount of epinephrine was significantly lower in the EGDT group (Fig. 3a), whereas norepinephrine was used less frequently in the noEGDT group without reaching statistical significance (Fig. 3b).

Cardiac TnT levels did not show statistically significant differences between the EGDT and noEGDT group (Fig. 4).

LDH at baseline, $2 \mathrm{~h}, 4 \mathrm{~h}$ and $8 \mathrm{~h}$ after ROSC showed no significant differences between both groups (Fig. 5).

Immediately after CPR, lactate concentrations increased up to $7-10 \mathrm{mmol} / \mathrm{l}$ in both groups. Comparison of lactate concentrations between 1 and $8 \mathrm{~h}$ exhibited a significant decrease to nearly baseline levels in the EGDT group ( $1 \mathrm{~h}$ vs $8 \mathrm{~h}$ ROSC: 7.9 vs. $1.7 \mathrm{mmol} / \mathrm{l}, P<0.01$ ), while in the noEGDT group lactate concentrations did not decrease significantly ( 1 h vs. 8 h ROSC: 9.1 vs. $3.8 \mathrm{mmol} / \mathrm{l}, P=0.06$; Fig. 6 ).

Total amount of fluids per therapeutic cycle displayed no significant difference between the two groups (EGDT $9.8 \pm 11.1 \mathrm{ml} / \mathrm{kg}$, noEGDT $7.6 \pm 10.4 \mathrm{ml} / \mathrm{kg}, P=0.259$; Supplement 2). With respect to target fluid administration, we recorded higher fluid administration in the initial $2 \mathrm{~h}$ after ROSC in EGDT animals (Supplement 2). Subanalysis of therapeutic cycles within the EGDT group demonstrated a significant higher volume loading compared to the noEGDT if the targets have been achieved (EGDT: $9.1 \pm 11.6 \mathrm{ml} / \mathrm{kg}$ vs noEGDT: $3.6 \pm 6.9 \mathrm{ml} /$ $\mathrm{kg}, P=0.026$; not shown).

\section{Discussion}

By using a randomized animal model, we could demonstrate that implementation of an EGDT algorithm after cardiac arrest and successful ROSC was in principle feasible. Furthermore, EGDT was associated with a more pronounced decrease of lactate concentrations to almost baseline levels and a significant improved systolic 


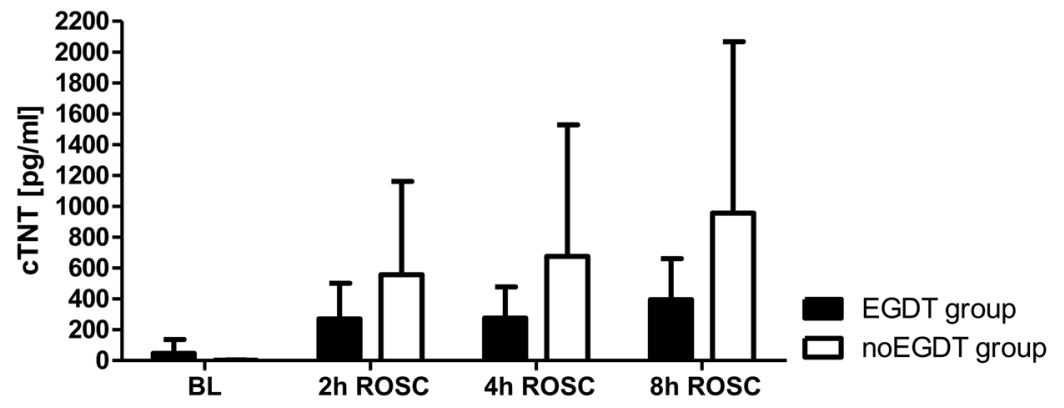

\begin{tabular}{|c|c|c|c|c|c|c|c|c|c|c|c|c|c|}
\hline & \multicolumn{7}{|c|}{$\begin{array}{l}\text { EGDT group }(\mathbf{N}=7) \\
\text { cTNT }[\mathrm{pg} / \mathrm{ml}]\end{array}$} & \multicolumn{6}{|c|}{$\begin{array}{l}\text { noEGDT group }(\mathbf{N}=\mathbf{6}) \\
\text { cTNT }[\mathrm{pg} / \mathrm{ml}]\end{array}$} \\
\hline BL & 250.1 & 3.0 & 3.0 & 3.0 & 35.0 & 4.7 & 27.7 & id & 3.0 & 4.9 & 3.0 & 3.0 & 3.0 \\
\hline 2h ROSC & 775.5 & 246.9 & 281.7 & 169.2 & 128.0 & 191.1 & 97.8 & 84.8 & 773.6 & 905.1 & 19.7 & 1505.0 & 49.0 \\
\hline 4h ROSC & 726.4 & 264.6 & 227.2 & 196.1 & 195.7 & 164.4 & 161.7 & 91.2 & 1042.0 & 662.8 & 21.1 & 2200.0 & 35.6 \\
\hline 8h ROSC & id & 917.4 & 402.1 & 185.3 & 280.1 & 306.4 & 277.6 & 185.9 & 1227.0 & 1425.0 & 21.5 & 2842.0 & 34.4 \\
\hline
\end{tabular}

Figure 4. Mean concentrations of plasma cTNT at baseline, $2 \mathrm{~h}, 4 \mathrm{~h}$ and $8 \mathrm{~h}$ after ROSC in the EGDT and noEGDT group (upper panel). Columns show the mean, bars denote SD. cTNT values for each animal are depicted in the table (lower panel). Id invalid data.

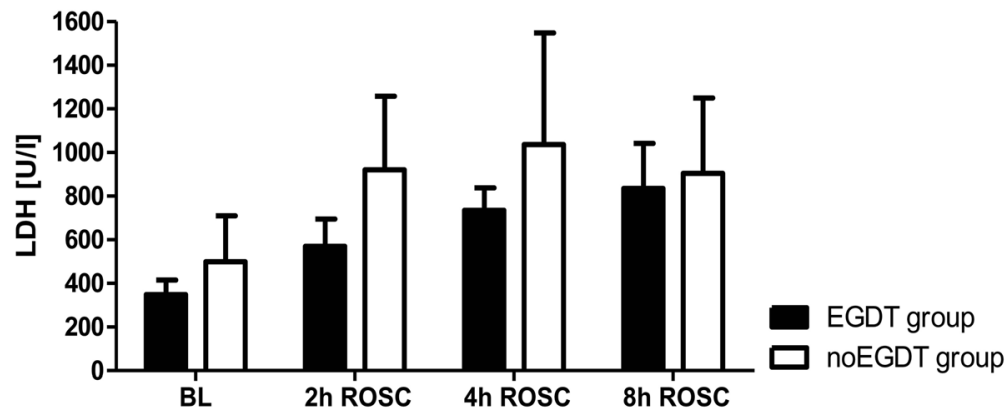

\begin{tabular}{|c|c|c|c|c|c|c|c|c|c|c|c|c|c|}
\hline \multirow[b]{2}{*}{ BL } & \multicolumn{7}{|c|}{$\begin{array}{c}\text { EGDT group }(\mathbf{N}=7) \\
\text { LDH }[\mathrm{U} / 1]\end{array}$} & \multicolumn{6}{|c|}{$\begin{array}{c}\text { noEGDT group }(\mathbf{N}=\mathbf{6}) \\
\text { LDH }[\mathrm{U} / \mathrm{l}]\end{array}$} \\
\hline & 285 & 278 & 426 & 274 & 417 & 378 & 382 & 246 & 216 & 620 & 688 & 608 & 621 \\
\hline 2h ROSC & 532 & 513 & 808 & 515 & 439 & 669 & 516 & 597 & 817 & 1505 & 835 & 1109 & 658 \\
\hline 4h ROSC & 874 & 779 & 732 & 657 & 553 & 784 & 765 & 564 & 868 & 1773 & 761 & 1587 & 664 \\
\hline 8h ROSC & 939 & 944 & 886 & 1148 & 553 & 757 & 621 & 531 & 990 & 1281 & 657 & 1325 & 640 \\
\hline
\end{tabular}

Figure 5. Mean catalytic activities of plasma LDH at baseline, $2 \mathrm{~h}, 4 \mathrm{~h}$ and $8 \mathrm{~h}$ after ROSC in the EGDT and noEGDT group (upper panel). Colunms show the mean, bars denote SD. Catalytic LDH activities for each animal are depicted in the table (lower panel).

myocardial function, which was not observed in the noEGDT group. The EGDT group revealed significant reduced need for epinephrine and the absence of hemodynamic life-threatening situations requiring immediate therapeutic intervention.

Although 50\% of initial successfully resuscitated patients die after administration to a hospital, there exist no evidence based guidelines for treatment of this specific group of patients ${ }^{4,5}$. A large proportion of deaths after cardiac arrest are caused by neurologic injur $y^{20}$. Therapeutic recommendations include percutaneous coronary intervention, target temperature management and goal directed hemodynamic optimization. However, performance and implementation of hemodynamic therapy after successful resuscitation is still elusive ${ }^{21}$. There are only few recommendations regarding hemodynamic post resuscitation care on which clinicians can orientate $e^{6,11,22,23}$. Rivers and colleagues have demonstrated that an early goal directed hemodynamic optimization could reduce mortality in septic patients ${ }^{24}$. In this context, a clinical study could demonstrate similarities in inflammatory response between septic patients and patients after cardiac arrest and successful ROSC ${ }^{25}$. Accordingly, other studies investigated the effect of an early goal directed hemodynamic optimization in patients after successful 


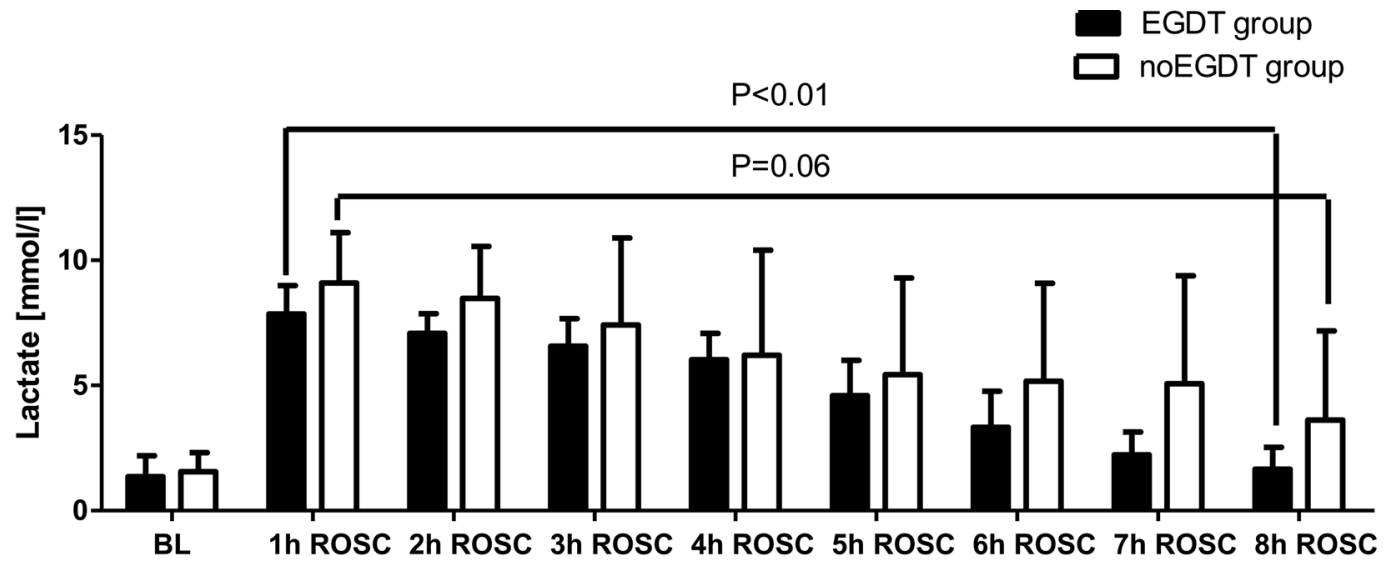

\begin{tabular}{|c|c|c|c|c|c|c|c|c|c|c|c|c|c|}
\hline \multirow[b]{2}{*}{ BL } & \multicolumn{7}{|c|}{$\begin{array}{l}\text { EGDT group }(\mathbf{N}=7) \\
\text { Lactate }[\mathrm{mmol} / 1]\end{array}$} & \multicolumn{6}{|c|}{$\begin{array}{c}\text { noEGDT group }(\mathbf{N}=\mathbf{6}) \\
\text { Lactate }[\mathrm{mmol} / 1]\end{array}$} \\
\hline & 1.20 & 1.60 & 2.80 & 2.00 & 0.60 & 0.60 & 0.70 & 2.30 & 1.90 & 0.80 & 2.30 & 0.50 & 1.50 \\
\hline 1h ROSC & 8.80 & 7.30 & 7.50 & 9.40 & 6.70 & 8.80 & 6.50 & 10.60 & 8.60 & 12.40 & 7.70 & 8.20 & 7.10 \\
\hline 2h ROSC & 6.70 & 7.70 & 7.10 & 7.60 & 7.30 & 7.70 & 5.50 & 10.60 & 9.20 & 8.80 & 10.10 & 5.00 & 7.20 \\
\hline 3h ROSC & 6.10 & 7.20 & 6.40 & 7.70 & 7.80 & 4.70 & 6.10 & 11.50 & 10.20 & 5.60 & 9.50 & 2.60 & 5.10 \\
\hline 4h ROSC & 4.70 & 6.10 & 4.80 & 7.40 & 7.10 & 5.60 & 6.50 & 11.20 & 10.60 & 3.50 & 7.70 & 1.50 & 2.70 \\
\hline 5h ROSC & 2.80 & 4.20 & 3.00 & 5.70 & 5.00 & 6.70 & 4.80 & 9.20 & 10.90 & 4.30 & 4.90 & 2.10 & 1.20 \\
\hline 6h ROSC & 2.00 & 3.60 & 1.90 & 4.60 & 2.90 & 5.80 & 2.50 & 5.70 & 11.80 & 6.80 & 2.30 & 3.60 & 0.80 \\
\hline 7h ROSC & id & 2.70 & 1.30 & 3.60 & 1.70 & 2.70 & 1.40 & 4.50 & 12.40 & 7.00 & 1.20 & 4.70 & 0.60 \\
\hline 8h ROSC & 2.00 & 2.30 & 0.90 & 3.20 & 1.10 & 1.10 & 1.00 & id & 9.00 & 5.40 & 0.80 & 2.20 & 0.70 \\
\hline
\end{tabular}

Figure 6. Mean concentrations of plasma lactate at baseline and between $1 \mathrm{~h}$ and $8 \mathrm{~h}$ after ROSC in the EGDT and noEGDT group (upper panel). Columns show the mean, bars denote SD. Lactate values for each animal are depicted in the table (lower panel). id invalid data.

resuscitation ${ }^{10,11,26}$. However, the relevance of these studies is weak due to implementation of EGDT within a therapeutic bundle.

Cardiac function. Because a potential beneficial impact of EGDT on cardiac function and myocardial damage after cardiac arrest was not studied systematically up to now, the aim of our study was to investigate these issues in an animal model.

We could demonstrate a positive impact of EGDT on systolic cardiac function in pigs after cardiac arrest and successful resuscitation. Thus, for example, PRSW as a pre- and afterload independent variable, EF and $\mathrm{dp} / \mathrm{dt}_{\max }$ as load-dependent parameters revealed significant higher values in the EGDT group compared to the noEGDT group. These observations were supported by the course of $\mathrm{S}_{\mathrm{cv}} \mathrm{O}_{2}$. This parameter is used to evaluate the ratio of oxygen supply and demand and also serves as an indirect marker for myocardial function. In our study, $\mathrm{S}_{\mathrm{cv}} \mathrm{O}_{2}$ in the EGDT group revealed a stable trend and no significant decrease over the time in contrast to the noEGDT group.

However, Ees as a parameter for cardiac contractility independent from pre- and afterload, exhibited no significant differences between both groups. Interestingly, after a considerable reduction caused by cardiac arrest, Ees demonstrated a continuous increase over time with reaching similar values at $8 \mathrm{~h}$ compared to baseline in the EGDT group. These findings were in accordance with other studies dealing with myocardial function after cardiac arrest. For example, Gazmuri et al. as one of a few investigated myocardial contractility in a cardiac arrest animal model by using a conductance catheter. The authors also observed a significant reduction in $\mathrm{Ees}^{27}$. Interestingly, in our study absolute values for EF were considerably higher compared to other investigations ${ }^{27,28}$. This could be explained by the absence of any early goal directed hemodynamic optimization in the aforementioned studies.

EGDT may solely be associated with improved myocardial function and hemodynamics ${ }^{29,30}$. For the assessment of diastolic myocardial function, we compared end-diastolic volume (EDV), Tau, $\mathrm{dp} / \mathrm{dt}_{\text {min }}$, EDPVR and end-diastolic pressure (EDP). Except for EDV, there were no significant differences between both groups. There are rare data concerning cardiac diastolic function after cardiac arrest. Kern and colleagues obtained an increased left ventricular end diastolic pressure (LVEDP) and Tau in domestic pigs following cardiac arrest ${ }^{28}$. In this context, another study dealing with survivors of out-of-hospital cardiac arrest reported an increased LVEDP in hemodynamic instable patients compared to the stable group ${ }^{31}$.

Hemodynamics and laboratory parameters. With respect to hemodynamic stabilization, recent studies recommended the usage of catecholamines in the post resuscitation period ${ }^{6,23,32}$. In daily clinical routine, 
MAP, lactate and urine output are often used for control of catecholamine therapy. Accordingly, low demand of cardiovascular drugs, especially epinephrine, seem to have positive impact on patient's outcome ${ }^{33,34}$.

It must be emphasized, however, that in our study the EGDT group exhibited significant lower doses of epinephrine. This might be explained by the differentiated therapy with volume and catecholamines in context of an early goal directed hemodynamic optimization. Several studies have shown, that despite of total amount of fluids, timing of fluid loading seems to play a more important role in hemodynamic optimization resulting in significant higher values for $\mathrm{CO}$ and $\mathrm{MAP}^{30,35}$. In this context, recent studies could demonstrate, that a target range of MAP between 80 and $100 \mathrm{mmHg}$ after cardiac arrest resulted in less myocardial injury. Furthermore, severe neurological dysfunction was associated with MAP thresholds $<75 \mathrm{mmHg}^{13,36-38}$.

With respect to myocardial damage after cardiac arrest, we could demonstrate that an early goal directed hemodynamic optimization in the post resuscitation period was associated with lower values for cTnT and LDH without reaching statistical significance. There are several causes associated with a troponin increase after cardiac arrest. One of the most common reasons is myocardial ischemia due to coronary artery stenosis or occlusion ${ }^{39}$. However, as we investigated young and healthy animals and ventricular fibrillation was electrically induced, coronary lesions appears unlikely. Other factors with influence on troponin release after cardiac arrest is the time required to reach ROSC and numbers of defibrillations ${ }^{40,41}$. With respect to our study, these two factors were negligible as we observed no significant differences between EGDT and noEGDT group. In this context, it must be noted that the differing standard deviations of duration of cardiopulmonary resuscitation in both groups were probably based on randomness and the small sample size. However, when looking at the distribution of the individual animals, it is noticeable that the distribution of cardiopulmonary resuscitation period in the noEGDT group was shifted towards shorter times. Therefore, the effect on myocardial ischemia cTNT may even be underestimated.

In our study, we observed the highest levels for lactate one hour after ROSC in both groups. There was a continuous decrease of lactate in both groups over the time, while only the EGDT group achieved similar concentrations after eight hours of ROSC compared to baseline. Elevation of serum lactate is a surrogate parameter of inadequate tissue perfusion and is accompanied with increased mortality and poor neurological outcome after cardiac arrest ${ }^{42,43}$. Several studies demonstrated a relationship between epinephrine and increased lactate levels in patients with septic shock ${ }^{44,45}$. As we observed significant lower total amounts of epinephrine in the EGDT group, this might have influenced our results for lactate concentrations.

Although there is a large proportion of deaths after cardiac arrest due to neurologic injury ${ }^{20}$, we did not measure biomarkers of neurologic injury in the present study due to methodological issues (partial occlusion of the carotid artery for conductance measurement). Therefore, we were unable to make a statement on the impact of hemodynamic optimization on neurologic injury after cardiac arrest. In this context, some investigations emphasized the use of regional cerebral oximetry or EEG derived variables during cardiopulmonary resuscitation and post-resuscitation period ${ }^{46,47}$. Early goal directed hemodynamic optimization after cardiac arrest seems capable of improving cerebral oxygenation. However, the extent of the influence on brain damage and neurological outcome remains elusive ${ }^{48}$.

As suggested by Gaieski and colleagues, the EGDT algorithm should be compared with therapies typically used in daily clinical routine ${ }^{14}$. In this context, it must be emphasized that there exist no concrete standards for post cardiac arrest care ${ }^{49}$. This seems to be one reason for the observed different mortality rates in various hospitals and regions indicating on different therapy approaches ${ }^{50}$. Therefore, in order to simulate these issues we used a dynamic concept for the noEGDT group.

Limitations. There are some limitations of our study that should be addressed. As we investigated healthy pigs with normal cardiac and pulmonary function, our results cannot directly transferred to patients after cardiac arrest exhibiting chronic diseases. Furthermore, we decided to treat the EGDT group by an algorithm in accordance to Gaieski and colleagues who used the CVP for guiding fluid therapy ${ }^{10}$. However, there are several confounding factors related to the predictive power of CVP. Thus, for example, CVP could be elevated in presence of cardiac tamponade, pulmonary embolism and myocardial infarction ${ }^{6}$. In our study we could rule out the influence of these confounding factors on the CVP by echocardiography. But, both the availably and examiners experience of echocardiography differs among various institutions. Finally, due to the exploratory character of the presented study and the high drop-out rates in both groups, this study might be under-powered.

\section{Conclusion}

In summary, the results of our study demonstrate the feasibility of an early goal directed hemodynamic optimization after cardiac arrest. EGDT is associated with a significant decrease of lactate levels to nearly baseline and is able to significantly improve systolic myocardial function. Additionally, the EGDT group shows a significantly reduced total amount of epinephrine. Although the results of our study suggest that implementation of an EGDT algorithm for post cardiac arrest care seems possible, the impact of algorithms with different hemodynamic parameters should be further investigated within clinical trials that are urgently needed.

Received: 7 May 2020; Accepted: 9 February 2021

Published online: 05 March 2021

\section{References}

1. Grasner, J. T. et al. Quality management in resuscitation-towards a European cardiac arrest registry (EuReCa). Resuscitation 82, 989-994. https://doi.org/10.1016/j.resuscitation.2011.02.047 (2011). 
2. Grmec, S. et al. Utstein style analysis of out-of-hospital cardiac arrest-bystander CPR and end expired carbon dioxide. Resuscitation 72, 404-414. https://doi.org/10.1016/j.resuscitation.2006.07.012 (2007).

3. Salcido, D. D., Stephenson, A. M., Condle, J. P., Callaway, C. W. \& Menegazzi, J. J. Incidence of rearrest after return of spontaneous circulation in out-of-hospital cardiac arrest. Prehosp. Emerg. Care Off. J. Natl. Assoc. EMS Phys. Natl. Assoc. State EMS Directors 14, 413-418. https://doi.org/10.3109/10903127.2010.497902 (2010).

4. Grasner, J. T. et al. Postresuscitation care with mild therapeutic hypothermia and coronary intervention after out-of-hospital cardiopulmonary resuscitation: A prospective registry analysis. Crit. Care 15, R61. https://doi.org/10.1186/cc10035 (2011).

5. Pell, J. P. et al. Presentation, management, and outcome of out of hospital cardiopulmonary arrest: Comparison by underlying aetiology. Heart 89, 839-842 (2003).

6. Neumar, R. W. et al. Post-cardiac arrest syndrome: Epidemiology, pathophysiology, treatment, and prognostication. A consensus statement from the International Liaison Committee on Resuscitation (American Heart Association, Australian and New Zealand Council on Resuscitation, European Resuscitation Council, Heart and Stroke Foundation of Canada, InterAmerican Heart Foundation, Resuscitation Council of Asia, and the Resuscitation Council of Southern Africa); the American Heart Association Emergency Cardiovascular Care Committee; the Council on Cardiovascular Surgery and Anesthesia; the Council on Cardiopulmonary, Perioperative, and Critical Care; the Council on Clinical Cardiology; and the Stroke Council. Circulation 118, 2452-2483. https://doi.org/10.1161/CIRCULATIONAHA.108.190652 (2008).

7. Kilgannon, J. H. et al. Association between arterial hyperoxia following resuscitation from cardiac arrest and in-hospital mortality. JAMA 303, 2165-2171. https://doi.org/10.1001/jama.2010.707 (2010).

8. Takasu, A., Saitoh, D., Kaneko, N., Sakamoto, T. \& Okada, Y. Hyperthermia: Is it an ominous sign after cardiac arrest?. Resuscitation 49, 273-277 (2001).

9. Russo, J. J. et al. Hyperglycaemia in comatose survivors of out-of-hospital cardiac arrest. Eur. Heart J. Acute Cardiovasc. Care 7, 442-449. https://doi.org/10.1177/2048872616684685 (2018).

10. Gaieski, D. F. et al. Early goal-directed hemodynamic optimization combined with therapeutic hypothermia in comatose survivors of out-of-hospital cardiac arrest. Resuscitation 80, 418-424. https://doi.org/10.1016/j.resuscitation.2008.12.015 (2009).

11. Sunde, K. et al. Implementation of a standardised treatment protocol for post resuscitation care after out-of-hospital cardiac arrest. Resuscitation 73, 29-39. https://doi.org/10.1016/j.resuscitation.2006.08.016 (2007).

12. Ameloot, K. et al. Hemodynamic targets during therapeutic hypothermia after cardiac arrest: A prospective observational study. Resuscitation 91, 56-62. https://doi.org/10.1016/j.resuscitation.2015.03.016 (2015).

13. Bhate, T. D., McDonald, B., Sekhon, M. S. \& Griesdale, D. E. Association between blood pressure and outcomes in patients after cardiac arrest: A systematic review. Resuscitation 97, 1-6. https://doi.org/10.1016/j.resuscitation.2015.08.023 (2015).

14. Gaieski, D. F. et al. Haemodynamic management strategies are not explicitly defined in the majority of therapeutic hypothermia implementation studies. Resuscitation 83, 835-839. https://doi.org/10.1016/j.resuscitation.2011.12.016 (2012).

15. Albrecht, M. et al. Evaluation of remote ischaemic post-conditioning in a pig model of cardiac arrest: A pilot study. Resuscitation 93, 89-95. https://doi.org/10.1016/j.resuscitation.2015.05.019 (2015).

16. Meybohm, P. et al. Pharmacological postconditioning with sevoflurane after cardiopulmonary resuscitation reduces myocardial dysfunction. Crit. Care 15, R241. https://doi.org/10.1186/cc10496 (2011).

17. Meybohm, P. et al. Hypothermia and postconditioning after cardiopulmonary resuscitation reduce cardiac dysfunction by modulating inflammation, apoptosis and remodeling. PLoS ONE 4, e7588. https://doi.org/10.1371/journal.pone.0007588 (2009).

18. Deakin, C. D. et al. European Resuscitation Council Guidelines for Resuscitation 2010 Section 4. Adult advanced life support. Resuscitation 81, 1305-1352. https://doi.org/10.1016/j.resuscitation.2010.08.017 (2010).

19. Idris, A. H. et al. Utstein-style guidelines for uniform reporting of laboratory CPR research. A statement for healthcare professionals from a task force of the American Heart Association, the American College of Emergency Physicians, the American College of Cardiology, the European Resuscitation Council, the Heart and Stroke Foundation of Canada, the Institute of Critical Care Medicine, the Safar Center for Resuscitation Research, and the Society for Academic Emergency Medicine. Writing Group. Writing Group. Circulation 94, 2324-2336 (1996).

20. Lemiale, V. et al. Intensive care unit mortality after cardiac arrest: The relative contribution of shock and brain injury in a large cohort. Intensive Care Med. 39, 1972-1980. https://doi.org/10.1007/s00134-013-3043-4 (2013).

21. Jones, A. E., Shapiro, N. I., Kilgannon, J. H. \& Trzeciak, S. Goal-directed hemodynamic optimization in the post-cardiac arrest syndrome: A systematic review. Resuscitation 77, 26-29. https://doi.org/10.1016/j.resuscitation.2007.10.021 (2008).

22. Peberdy, M. A. et al. Part 9: Post-cardiac arrest care: 2010 American Heart Association Guidelines for Cardiopulmonary Resuscitation and Emergency Cardiovascular Care. Circulation 122, S768-786. https://doi.org/10.1161/CIRCULATIONAHA.110.971002 (2010).

23. Nolan, J. P. et al. European Resuscitation Council and European Society of Intensive Care Medicine Guidelines for Post-resuscitation Care 2015: Section 5 of the European Resuscitation Council Guidelines for Resuscitation 2015. Resuscitation 95, 202-222. https://doi.org/10.1016/j.resuscitation.2015.07.018 (2015).

24. Rivers, E. et al. Early goal-directed therapy in the treatment of severe sepsis and septic shock. N. Engl. J. Med. 345, 1368-1377 (2001).

25. Adrie, C. et al. Successful cardiopulmonary resuscitation after cardiac arrest as a "sepsis-like" syndrome. Circulation 106, 562-568 (2002).

26. Walters, E. L. et al. Implementation of a post-cardiac arrest care bundle including therapeutic hypothermia and hemodynamic optimization in comatose patients with return of spontaneous circulation after out-of-hospital cardiac arrest: A feasibility study. Shock 35, 360-366. https://doi.org/10.1097/SHK.0b013e318204c106 (2011).

27. Gazmuri, R. J. et al. Myocardial dysfunction after successful resuscitation from cardiac arrest. Crit. Care Med. 24, 992-1000 (1996).

28. Kern, K. B., Hilwig, R. W., Rhee, K. H. \& Berg, R. A. Myocardial dysfunction after resuscitation from cardiac arrest: An example of global myocardial stunning. J. Am. Coll. Cardiol. 28, 232-240 (1996).

29. Jacobshagen, C. et al. Effects of mild hypothermia on hemodynamics in cardiac arrest survivors and isolated failing human myocardium. Clin. Res. Cardiol. Off. J. German Cardiac Soc. 99, 267-276. https://doi.org/10.1007/s00392-010-0113-2 (2010).

30. Broch, O. et al. Non-invasive hemodynamic optimization in major abdominal surgery: A feasibility study. Minerva Anestesiol. 82, 1158-1169 (2016).

31. Laurent, I. et al. Reversible myocardial dysfunction in survivors of out-of-hospital cardiac arrest. J. Am. Coll. Cardiol. 40, 2110-2116 (2002).

32. Huang, L., Weil, M. H., Tang, W., Sun, S. \& Wang, J. Comparison between dobutamine and levosimendan for management of postresuscitation myocardial dysfunction. Crit. Care Med. 33, 487-491 (2005).

33. Beylin, M. E. et al. Higher mean arterial pressure with or without vasoactive agents is associated with increased survival and better neurological outcomes in comatose survivors of cardiac arrest. Intensive Care Med. 39, 1981-1988. https://doi.org/10.1007/ s00134-013-3075-9 (2013).

34. Wang, H. E., Min, A., Hostler, D., Chang, C. C. \& Callaway, C. W. Differential effects of out-of-hospital interventions on shortand long-term survival after cardiopulmonary arrest. Resuscitation 67, 69-74. https://doi.org/10.1016/j.resuscitation.2005.05.008 (2005). 
35. Salzwedel, C. et al. Perioperative goal-directed hemodynamic therapy based on radial arterial pulse pressure variation and continuous cardiac index trending reduces postoperative complications after major abdominal surgery: A multi-center, prospective, randomized study. Crit. Care 17, R191. https://doi.org/10.1186/cc12885 (2013).

36. Ameloot, K. et al. Optimum blood pressure in patients with shock after acute myocardial infarction and cardiac arrest. J. Am. Coll. Cardiol. 76, 812-824. https://doi.org/10.1016/j.jacc.2020.06.043 (2020).

37. Russo, J. J. et al. Impact of mean arterial pressure on clinical outcomes in comatose survivors of out-of-hospital cardiac arrest: Insights from the University of Ottawa Heart Institute Regional Cardiac Arrest Registry (CAPITAL-CARe). Resuscitation 113, 27-32. https://doi.org/10.1016/j.resuscitation.2017.01.007 (2017).

38. Russo, J. J. et al. Optimal mean arterial pressure in comatose survivors of out-of-hospital cardiac arrest: An analysis of area below blood pressure thresholds. Resuscitation 128, 175-180. https://doi.org/10.1016/j.resuscitation.2018.04.028 (2018).

39. Thygesen, K. et al. Third universal definition of myocardial infarction. J. Am. Coll. Cardiol. 60, 1581-1598. https://doi.org/10. 1016/j.jacc.2012.08.001 (2012).

40. Mullner, M. et al. The influence of chest compressions and external defibrillation on the release of creatine kinase-MB and cardiac troponin $\mathrm{T}$ in patients resuscitated from out-of-hospital cardiac arrest. Resuscitation 38, 99-105 (1998).

41. Lin, C. C., Chiu, T. F., Fang, J. Y., Kuan, J. T. \& Chen, J. C. The influence of cardiopulmonary resuscitation without defibrillation on serum levels of cardiac enzymes: A time course study of out-of-hospital cardiac arrest survivors. Resuscitation 68, 343-349. https://doi.org/10.1016/j.resuscitation.2005.07.018 (2006).

42. Donnino, M. W. et al. Initial lactate and lactate change in post-cardiac arrest: A multicenter validation study. Crit. Care Med. 42, 1804-1811. https://doi.org/10.1097/CCM.0000000000000332 (2014).

43. Dell'Anna, A. M. et al. Prognostic implications of blood lactate concentrations after cardiac arrest: A retrospective study. Ann. Intensive Care 7, 101. https://doi.org/10.1186/s13613-017-0321-2 (2017).

44. Levy, B. et al. Comparison of norepinephrine and dobutamine to epinephrine for hemodynamics, lactate metabolism, and gastric tonometric variables in septic shock: A prospective, randomized study. Intensive Care Med. 23, 282-287 (1997).

45. Day, N. P. et al. The effects of dopamine and adrenaline infusions on acid-base balance and systemic haemodynamics in severe infection. Lancet 348, 219-223. https://doi.org/10.1016/s0140-6736(96)09096-4 (1996).

46. Genbrugge, C. et al. Regional cerebral oximetry during cardiopulmonary resuscitation: Useful or useless?. J. Emerg. Med. 50, 198-207. https://doi.org/10.1016/j.jemermed.2015.03.043 (2016).

47. Eertmans, W. et al. The prognostic value of bispectral index and suppression ratio monitoring after out-of-hospital cardiac arrest: A prospective observational study. Ann. Intensive Care 8, 34. https://doi.org/10.1186/s13613-018-0380-z (2018).

48. Ameloot, K. et al. Early goal-directed haemodynamic optimization of cerebral oxygenation in comatose survivors after cardiac arrest: the Neuroprotect post-cardiac arrest trial. Eur. Heart J. 40, 1804-1814. https://doi.org/10.1093/eurheartj/ehz120 (2019).

49. Gaieski, D. F., Abella, B. S. \& Goyal, M. CPR and postarrest care: Overview, documentation, and databases. Chest 141, $1082-1089$. https://doi.org/10.1378/chest.11-2130 (2012).

50. Nichol, G. et al. Regional variation in out-of-hospital cardiac arrest incidence and outcome. JAMA 300, 1423-1431. https://doi. org/10.1001/jama.300.12.1423 (2008).

\section{Acknowledgements}

The authors like to thank Dr. Tobias Neumann, Dr. Tobias Drews, Dr. Timo Iden, Isabelle Hartramph und Dr. Christoph Lauenstein for excellent technical assistance and support.

\section{Author contributions}

All authors contributed in the design of the paper and approved the final version of the manuscript. Study concept and design: O.B., J.R., P.M., M.S., B.B., M.G. Practical implementation of experiments: J.R., P.R., A.C.R., P.M., M.G. Data analyses and statistical analyses: O.B., J.R., P.R., A.C.R., M.G. Writing of the manuscript: O.B., M.G., M.A., L.H. Critical revision of the manuscript: M.A., L.H.

\section{Funding}

This work was supported by the Department of Anesthesiology and Operative Intensive Care Medicine, University Hospital Schleswig-Holstein, Campus Kiel. There were no commercial funding sources supporting the submitted study. Open Access funding enabled and organized by Projekt DEAL

\section{Competing interests}

JR has received honoraria from Edwards Lifesciences (Irvine, CA, USA) for giving lectures. BB has received honoraria from Pulsion Medical Systems (Feldkirchen, Germany) and Edwards Lifesciences (Irvine, CA, USA) for giving lectures. MG has received honoraria from GE Healthcare (Helsinki, Finland) and Pulsion Medical Systems (Feldkirchen, Germany) for giving lectures. No other author has a conflict of interest with regard to any device employed in this study.

\section{Additional information}

Supplementary Information The online version contains supplementary material available at https://doi.org/ 10.1038/s41598-021-83925-3.

Correspondence and requests for materials should be addressed to L.H.

Reprints and permissions information is available at www.nature.com/reprints.

Publisher's note Springer Nature remains neutral with regard to jurisdictional claims in published maps and institutional affiliations. 
(c) (i) Open Access This article is licensed under a Creative Commons Attribution 4.0 International cc) License, which permits use, sharing, adaptation, distribution and reproduction in any medium or format, as long as you give appropriate credit to the original author(s) and the source, provide a link to the Creative Commons licence, and indicate if changes were made. The images or other third party material in this article are included in the article's Creative Commons licence, unless indicated otherwise in a credit line to the material. If material is not included in the article's Creative Commons licence and your intended use is not permitted by statutory regulation or exceeds the permitted use, you will need to obtain permission directly from the copyright holder. To view a copy of this licence, visit http://creativecommons.org/licenses/by/4.0/.

(C) The Author(s) 2021 